\title{
Diagnosis and etiological identification in severe constrictive pericarditis in a 14-year-old girl: a case report
}

\author{
Lei Huang ${ }^{1,2}$, Quan Zhou ${ }^{1,2}$, Xiaoqiong Cui ${ }^{1,2}$, Xiaomin Hu${ }^{1,2}$ Dawei Duan ${ }^{1,2}$, Peng Wu ${ }^{1,2}$, Wenqing Gao ${ }^{1,2}$, \\ Meng Ning ${ }^{1,2}$, Tong $\mathrm{Li}^{1,2}$ \\ ${ }^{1}$ Department of Heart Center, Tianjin Third Central Hospital, Tianjin, China; ${ }^{2}$ Tianjin Key Laboratory of Extracorporeal Life Support for Critical \\ Diseases, Tianjin, China \\ Correspondence to: Professor Tong Li. Department of Heart Center, Tianjin Third Central Hospital, Tianjin Key Laboratory of Extracorporeal Life \\ Support for Critical Diseases, 83 Jintang Road, Hedong District, Tianjin, China. Email: litong3zx@sina.com.
}

\begin{abstract}
Constrictive pericarditis in children is exceedingly rare, and may cause very problematic confusion of diagnosis and etiology identification. In this case, we examined a 14-year-old female patient who had developed signs of significant anasarca which was eventually turned out to be constrictive pericarditis. Affected by the experience of examiners, the patient was not diagnosed or even suspected with constrictive pericarditis when she was initially examined by echocardiography in the hospital where she visited before. Reexamination of echocardiography, cardiac catheterization and non-invasive image techniques were performed to establish the diagnosis finally. Open pericardectomy was ultimately performed and normal hemodynamic parameters and cardiac function were obtained postoperatively. In the determination of etiology, we inferred that chronic infection induced by local virus infection in the pericardium led to constrictive pericarditis. Parvovirus B19 (PVB19) and/or human herpes virus 6 (HHV-6) were the two most likely viruses involved based on published literature reviews. Importantly, we learned that serological antibody testing may be false-negative and polymerase chain reaction (PCR) or metagenomic nextgeneration sequencing for pericardial viral nucleic acid testing may be the gold standard for confirmation. Unfortunately, fresh pericardial tissue samples were not taken before paraformaldehyde fixation in our case, which made it impossible for us to detect suspicious viruses. We do hope that the lessons learned from this case will be helpful and instructive for the etiological diagnosis of similar patients in the future.
\end{abstract}

Keywords: Constrictive pericarditis (CP); viral infection; child; heart failure; case report

Submitted Jul 21, 2020. Accepted for publication Oct 27, 2020.

doi: $10.21037 /$ tp-20-203

View this article at: http://dx.doi.org/10.21037/tp-20-203

\section{Introduction}

Constrictive pericarditis (CP) in children is exceedingly rare, and may cause very problematic confusion of diagnosis (1). Timely and accurate recognition of CP and early surgical intervention are always life-saving. On the other hand, etiology identification can be really challenging in clinical practice, which was attributed to the fact that children are less likely than adults to have a known set of etiological diagnoses, like tuberculosis or tumor. It has been reported that almost half of the cases of CP the etiology remains unknown, and the cases were considered idiopathic (2).
In this case, we examined a 14-year-old girl who was first admitted to our digestive system department with significant anasarca and a negative echocardiogram result diagnosed by another hospital. Cardiac catheterization and non-invasive image techniques were performed to establish the diagnosis. Pericardectomy was ultimately performed. Normal hemodynamic parameters and cardiac function were obtained postoperatively. Chronic infection of the localized pericardial parvovirus B19 and/or human herpes virus 6 is a potential cause of CP based on published literature reviews. From this case, we learned that the importance of recognizing certain common but easily 
neglected viral infections when pursuing CP etiology, especially in children serological antibody testing may be false-negative and nesting polymerase chain reaction or metagenomic next-generation sequencing for pericardial viral nucleic acid testing might be the gold standard when confirming suspected pathogens. We present the following case in accordance with the CARE reporting checklist (available at: http://dx.doi.org/10.21037/tp-20-203).

\section{Case presentation}

A previously fit, 14-year-old girl was admitted with complaints of anasarca and exercise intolerance (no shortness of breath at rest) that had been progressively aggravated for six months. Abdominal ultrasound and CT scan performed outside the hospital revealed evident hepatosplenomegaly, congested hepatic veins, multiple enlarged lymph nodes located around the mesenteric root, retroperitoneal, and inguinal regions, and no ascites was found; the echocardiography showed no obvious abnormal signs apart from decreased diastolic function. The patient was first admitted to our digestive system department, where she denied any history of tuberculosis, infectious disease, or epidemic contact.

Physical examination showed body weight of 52 kilograms, temperature of 36.7 degrees celsius, 68 beat $/ \mathrm{min}$ heart rates, 100/70 mmHg non-invasive blood pressure, 18 per minute of respiratory rate and oxygen saturation $98 \%$ on room air. The inferior border of the liver was able to be palpitated under the costal arch by $4 \mathrm{~cm}$, and lower limbs showed obvious pitting edema. A second echocardiography, which was performed by an experienced doctor in our center, revealed thickened pericardium (with the thickest point at approximately $3.9 \mathrm{~mm}$ ) but no calcification, as well as dilated atria, relatively small ventricles with normal systolic left-ventricular function (LVEF 61\%), decreased biventricular diastolic function, and bounced inter-ventricular septum. There was no observable pericardial effusion (Figure 1A). Laboratory studies yielded normal hepatic and renal function except for mild elevation of gammaglutamyl transferase and total bilirubin, with no blood count, electrolyte, B-type natriuretic peptide, myocardial enzyme, erythrocyte sedimentation rate, tumor marker, thyroid function, or sex hormone level abnormalities. There likewise were no signs of any autoimmune process, and tuberculosis was ruled out by anti-tuberculosis antibody and tuberculin test. Serological results did not indicate acute infection with a group of common viruses, which included cytomegalovirus, adenovirus, Epstein-Barr virus, respiratory syncytial virus and Coxsackie virus. CP was suspected, so the patient was transferred to our center for further evaluation and treatment.

To complete the diagnostic work-up, cardiac magnetic resonance imaging scan was performed. In accordance with the echocardiographic observations, a dense mass surrounding nearly the entire heart was evident in addition to diastolic dysfunction and dilatation of both atria and central veins (Figure 1B,C). Next, cardiac catheterization was performed for confirmation and hemodynamics assessment. Mean atrial pressure and end-diastolic ventricular pressure were increased and almost equal in both atria and ventricles (right atrial pressure was $25 \mathrm{mmHg}$, right ventricle diastolic pressure was $17 \mathrm{mmHg}$, and left ventricle diastolic pressure was $16 \mathrm{mmHg}$ ), suggesting constrictive pathophysiology. A CT scan of the chest, a regular preoperative assessment tool in our center, yielded similar results as MRI (Figure 1D).

Constrictive pericarditis (CP) diagnosis was established and pericardectomy was performed. During surgery, operators found a massive adhesion of the diffusely thickened, tough pericardium. As the heart itself was firmly encased at the anterior wall, the parietal pericardium was incised with multiple "crossover" incisions, decompressed, and gradually stripped under cardiopulmonary bypass (Figure 2A). After transection of the thickened pericardium across the center of the right ventricle, the central venous pressure suddenly decreased by $10 \mathrm{mmHg}$. At the end of the surgical procedure, the central venous pressure had stayed within the range of $10-11 \mathrm{mmHg}$. Liver function was recovered gradually postoperatively, and the patient was discharged in good health. She then stayed free of symptoms and had a normal echocardiography at a follow-up visit five month later. Histopathologic examination of the resected pericardium showed extensive hyaline degeneration of the extracellular matrix, scattered lymphocytes, granulocyte infiltration, congested small vessels, and a large amount of vascular extravasation of erythrocytes where hemosiderin cells were identified. We also were surprised to find a pathophysiological change mimicking primitive chondroid tissue in some parts, which had not been previously reported (Figure 2B,C,D). The timeline of the case was demonstrated in Figure 3.

All procedures performed in studies involving human participants were in accordance with the ethical standards of the institutional and national research committee and with the Helsinki Declaration (as revised in 2013). Written informed consent was obtained from the patient. 

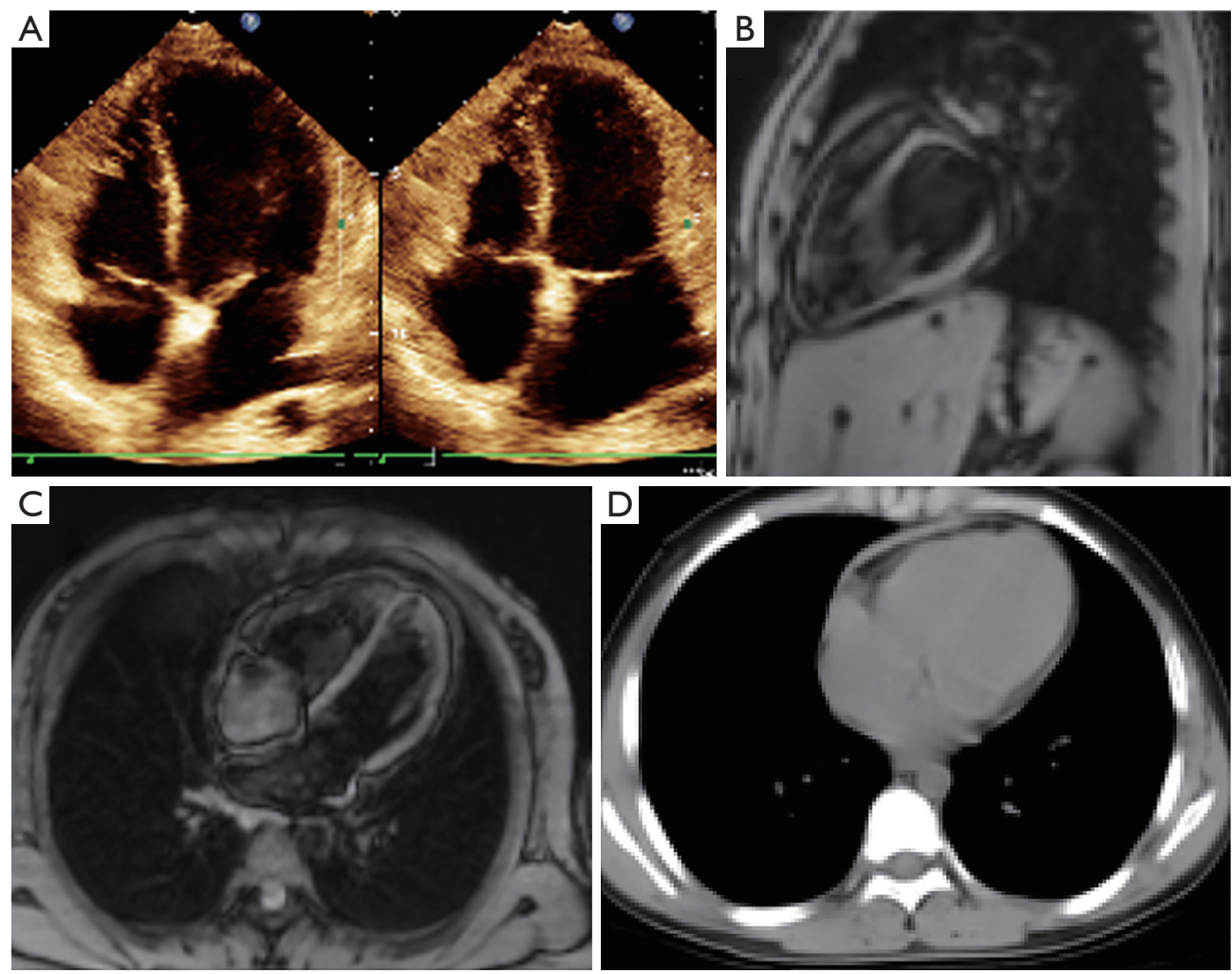

Figure 1 The imaging data used in the diagnosis of the patient. (A) Echocardiography (four-chamber view) demonstrates enlarged atria and pulmonary vein, relatively small ventricles; (B) the sagittal section (B) and transverse section (C) of cardiac MRI shows a dense and thickened percardium surrounding nearly the entire heart, as well as diastolic dysfunction, dilated atria and relatively small ventricles; (D) the chest CT scan shows a similar result as MRI. CT, computed tomography; MRI, magnetic resonance imaging.

\section{Discussion}

Constrictive pericarditis (CP) is an exceedingly rare but very serious illness in children, and is a challenging condition to diagnose (1). The illness presents various symptoms, making any diagnosis based only on clinical history impossible; further, some patients with CP may show false-negative findings on chest $\mathrm{X}$-rays. It is worth noting that $\mathrm{CP}$ and restrictive cardiomyopathy have similar clinical features to some extent. In this case, accurate echocardiography evaluation plays a vital role in the early identification of CP. Restrictive cardiomyopathy mainly involves the left ventricle, with signs of left atrial enlargement, pulmonary vein congestion while the right ventricular pulse is more obvious, thus the sign of vena cava congestion is not obvious. In contrast, both left and right ventricular pulsation are attenuated and there's no sign of atrial enlargement in CP. Another important distinction is that the pericardium of restrictive cardiomyopathy is normal whereas the cardiac chambers are usually enlarged, sometimes accompanied by endocardial thickening and mural thrombus. In contrast, CP is usually characterized by thickened or calcified pericardium.

In the patient examined in this study, mildly thickened pericardium without calcification and typical symptoms caused us to suspect CP. Then cardiac catheterization confirmed the diagnosis. It is important to note, though, that false-positive findings on CT, such as focal or diffuse thickening of the pericardium and dilated inferior vena cava, can also be found in patients without CP (3).

Although valuable non-invasive and invasive diagnostic tools provide logical and reliable information for CP diagnosis, a sizable challenge posed in clinical practice is successfully identifying its etiology-especially in children, who are most likely to lack a wide spectrum of well-known etiologies compared to adults. The child patient in this case had never been seriously ill before, and thus lacked any known spectrum of etiologies even after extensive inquiry into her history, including laboratory examination 

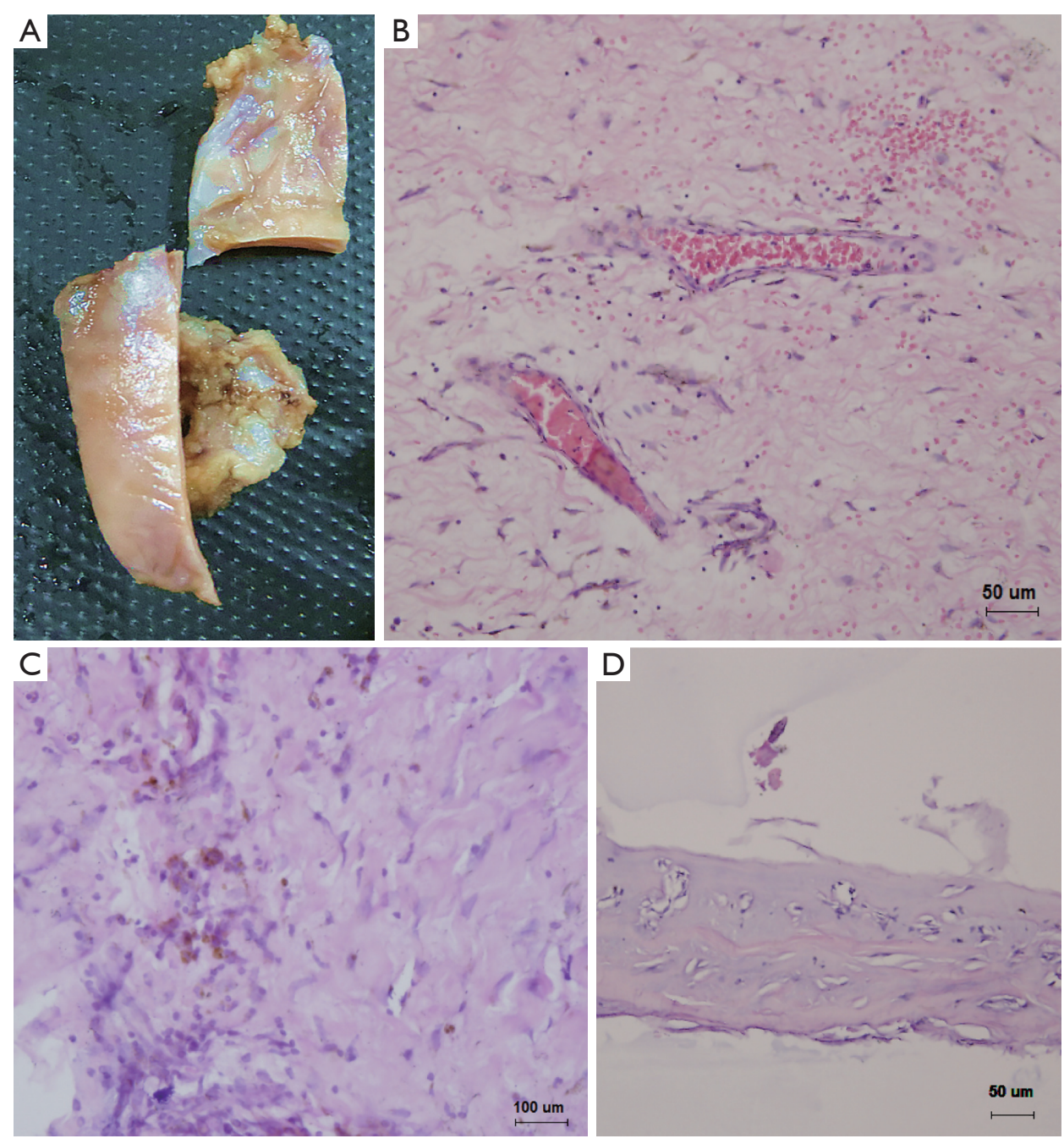

Figure 2 The gross and pathological characteristics of pericardial tissue resected. (A) Portions of resected tough pericardium with crossover incision, thickened up to 3-4 mm. (B) Hematoxylin-eosin (HE) staining of the pericardium, scattered lymphocytes, granulocytes infiltration, congested small vessels, and abundant vascular extravasation of erythrocytes (HE $\times 200)$. (C) Extensive hyaline degeneration of extracellular matrix, where hemosiderin cells were identified $(\mathrm{HE} \times 400)$. (D) Pathophysiological change mimicking primitive chondroid tissue in pericardium $(\mathrm{HE} \times 200)$.

and image studies. After taking lymphadenopathy into account, we suspected the patient's clinical symptoms may be compatible with primary virus infections. Quite disappointingly, however, the tests for immunoglobulin antibodies of the multiple common viruses which were mentioned in the case presentation part, were all negative.

After finding a rare case report of severe CP after parvovirus B19 (PVB19) and human herpes virus 6 (HHV-6) infection in a 9-year-old girl (4), as well as a study by Rohayem et al. on an 11-year-old male patient with fatal myocarditis due to parvovirus B19 in the course of a concomitant HHV-6 infection (5), we adjusted our approach. In the latter case, the authors obtained negative results either from the virus detected by immunoglobulin antibodies or from bacterial cultures or virus isolation from body fluids. Instead, the genomes of both viruses were eventually identified by nested polymerase chain reaction (PCR) within the tissue samples involved (resected pericardium), leading the authors of both articles to hypothesize that local HHV6-induced immunosuppression enhanced PVB19 infection and dissemination, thus resulting in acute fatal myocarditis, or chronic infection 


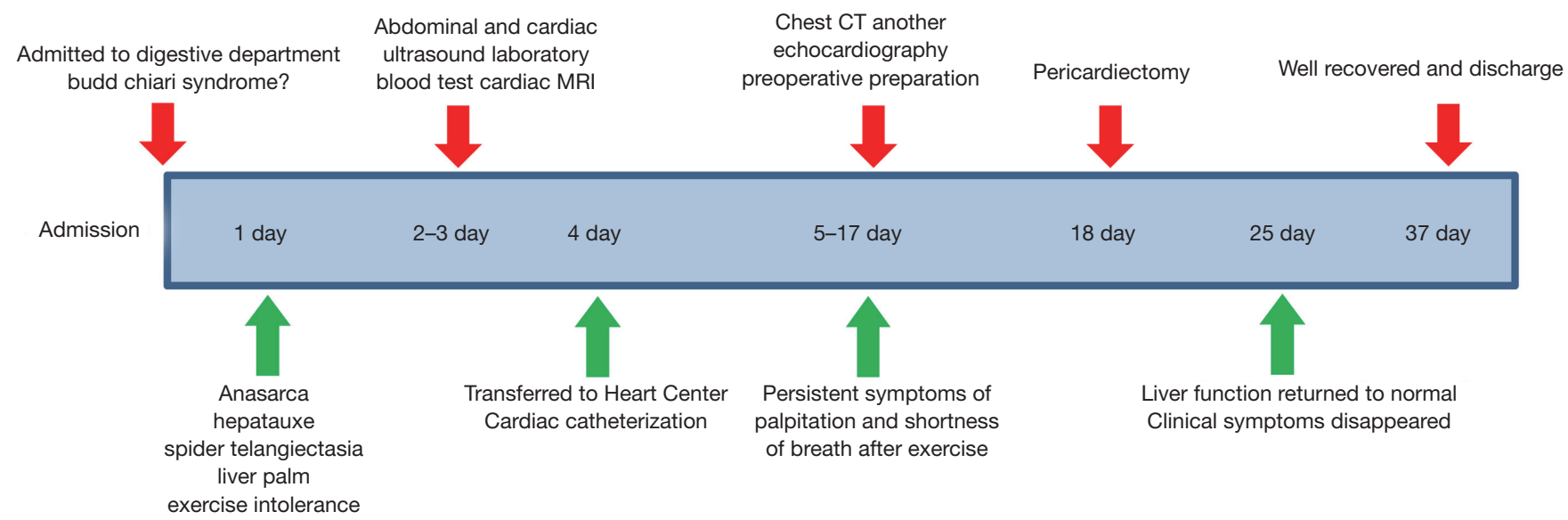

Figure 3 Timeline of the case.

leading to CP. As such, our negative serological results can be explained by the absence of the above viral infectionsor, alternately, that our serological tests did not accurately identify any viral infection. Because the pericardial tissue sample in our case was treated with formaldehyde, PCR detection of suspicious viruses was not possible.

Both parvovirus B19 and HHV-6 are ubiquitous viruses that can cause mild diseases in childhood. PB19 infection has been reported to be a rare but severe cause of myocarditis in infants and children (5), and HHV-6 is the causative agent of exanthema subitum. In our case, the patient's mother gave an account of childhood diseases that included exanthema subitum when the patient was 1-2 years of age, indicating a history of HHV-6 infection; it remains unknown whether a causal relationship exists between the onset of acute viral infection and CP morbidity, however. The sudden onset of clinical symptoms, which did not last longer than six months before the initial presentation, does not exclude a silent, long-term process.

Although quite unusual, this case report highlights the importance of recognizing certain common but easily neglected viral infections when pursuing $\mathrm{CP}$ etiology of $\mathrm{CP}$ in children. Importantly, serological antibody testing may be false-negative and polymerase chain reaction (PCR) or metagenomic next-generation sequencing (6) for pericardial viral nucleic acid testing may be the gold standard for confirmation.

\section{Acknowledgments}

Funding: This work was supported by the grant from the Tianjin Third Central Hospital National Natural Science
Foundation Incubation Project of China (Grant number 2019YNR1) and the Natural Science Foundation of Tianjin (Grant No. 19JCYBJC26000).

\section{Footnote}

Reporting Checklist: The authors have completed the CARE reporting checklist. Available at http://dx.doi.org/10.21037/ tp-20-203

Conflicts of Interest: All authors have completed the ICMJE uniform disclosure form (available at http://dx.doi. org/10.21037/tp-20-203). The authors have no conflicts of interest to declare.

Etbical Statement: The authors are accountable for all aspects of the work in ensuring that questions related to the accuracy or integrity of any part of the work are appropriately investigated and resolved. All procedures performed in studies involving human participants were in accordance with the ethical standards of the institutional and national research committee and with the Helsinki Declaration (as revised in 2013). Written informed consent was obtained from the patient.

Open Access Statement: This is an Open Access article distributed in accordance with the Creative Commons Attribution-NonCommercial-NoDerivs 4.0 International License (CC BY-NC-ND 4.0), which permits the noncommercial replication and distribution of the article with the strict proviso that no changes or edits are made and the original work is properly cited (including links to both the 
formal publication through the relevant DOI and the license). See: https://creativecommons.org/licenses/by-nc-nd/4.0/.

\section{References}

1. Troughton RW, Asher CR, Klein AL. Pericarditis. Lancet 2004;363:717-27.

2. Bertog SC, Thambidorai SK, Parakh K, et al. Constrictive pericarditis: etiology and cause-specific survival after pericardiectomy. J Am Coll Cardiol 2004;43:1445-52.

3. Chen SJ, Li YW, Wu MH, et al. CT and MRI Findings in a Child with Constrictive Pericarditis. Pediatr Cardiol 1998;19:259-62.

Cite this article as: Huang $\mathrm{L}$, Zhou Q, Cui X, Hu X, Duan D, Wu P, Gao W, Ning M, Li T. Diagnosis and etiological identification in severe constrictive pericarditis in a 14-yearold girl: a case report. Transl Pediatr 2021;10(1):209-214. doi: $10.21037 /$ tp-20-203
4. Backhoff D, Steinmetz M, Ruschewski W, et al. Severe Constrictive Pericarditis After Parvovirus B19 and Human Herpes Virus 6 Infection in a 9-Year-Old Girl. Pediatr Cardiol 2013;34:2089-92.

5. Rohayem J, Dinger J, Fischer R, et al. Fatal Myocarditis Associated with Acute Parvovirus B19 and Human Herpesvirus 6 Coinfection. J Clin Microbiol 2001;39:4585-7.

6. Miao Q, Ma Y, Wang Q, et al. Microbiological Diagnostic Performance of Metagenomic Next-generation Sequencing When Applied to Clinical Practice. Clin Infect Dis 2018;67:S231-40. 